\title{
Non-Stress Test in High-Risk Pregnancy
}

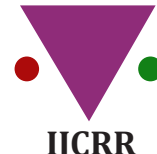

Section: Healthcare

Sci. Journal Impact

Factor: 6.1 (2018)

ICV: 90.90 (2018)

\section{R. P. Patange, S. S. Patil, Prashant D. Shah, Prashant D. Shah, Digvijiay Kadam, Vrishali Chavan}

Department of Ob/Gyn, Krishna Institute of Medical Sciences Deemed To Be University, Karad India.

\section{ABSTRACT}

Introduction: The journey of the foetus through the maternal pelvis is considered most difficult. The art of obstetrics is based on balancing the risks encountered during this journey.

Aims and Objectives: To evaluate the relation between the result of the non-stress test and mode of delivery. To evaluate the relation between the result of the non stress test and perinatal outcome.

This prospective study was carried out in the Department of Obstetrics and Gynecology at Krishna Institute of Medical Sciences, Karad. All high-risk patients admitted for induction of labour or in the latent phase of One hundred and fifty patients with highrisk pregnancies like pregnancy-induced hypertension, intrauterine growth retardation, overdue pregnancy, oligohydramnios, gestational diabetes and heart disease. Results- Patients with the gestational age of more than 34 weeks were subjected to the non-stress test. Out of 150 patients, 85 patients (56.67\%) had normal, 44 patients (29.33\%) had suspicious and 21 patients (14\%) had abnormal non-stress test results. $80 \%$ of patients with normal non-stress test deliver vaginally as against $50 \%$ in suspicious group and $28.57 \%$ in abnormal group. Caesarean section rate in the normal group was $20 \%$ while that in the suspicious group was $50 \%$ and $71.43 \%$ in abnormal group. In the abnormal group, caesarean section was done in $73.33 \%$ for cause related to the result of a non stress test.

Conclusion: Non-stress test is a simple, non-invasive, inexpensive test for antepartum foetal surveillance. It is easy to perform and causes no inconvenience or complications to the patient. The non-stress test should be performed for diagnostic purpose in the light of clinical circumstances.

Key Words: Non-Stress Test, High Risk, Pregnancy.

\section{INTRODUCTION}

With the emphasis on "Small Family Norm", it is all the more necessary that every wanted conception should end in the birth of a viable healthy baby. For this, close monitoring for assessment of foetal wellbeing is required especially for high-risk pregnancies. ${ }^{1}$ The ability to predict foetal survival for a finite interval has a major implication for both mother and foetus. The journey of the foetus through the maternal pelvis is considered most difficult. The art of obstetrics is based on balancing the risks encountered during this journey. For high-risk pregnancies, this balance is usually between conservative management with the risks of stillbirth and intervention with risks of prematurity complications and increased probability of operative delivery. The management of high-risk patient has always been a difficult and challenging problem. Good antenatal services backed up by vigilant intrapartum monitoring for the progress of labour and foetal conditions are the mainstay in the management of high-risk pregnancies.

A non-stress test is one of the biophysical technique for assessing the foetal condition and is one of the mainstays in the management of most high-risk pregnancies. It can identify the foetus in jeopardy, in the compromised intrauterine environment and also the foetus that may not be able to tolerate the stress of labour. ${ }^{2}$ This enables an appropriate and timely intervention to achieve the most favourable neonatal outcome.

\section{Corresponding Author:}

Dr. S. S. Patil, Department of Ob/Gyn, Krishna Institute of Medical Sciences Deemed To Be University, Karad India Email: hodobgyn@kimsuniversity.in

ISSN: 2231-2196 (Print)

Received: 12.07 .2020
ISSN: $0975-5241$ (Online)

Revised: 20.08 .2020
Accepted: 06.09.2020
Published: 22.09 .2020 


\section{AIMS AND OBJECTIVES}

1. To evaluate the relation between the result of the nonstress test and mode of delivery.

2. To evaluate the relation between the result of the non stress test and perinatal outcome.

\section{MATERIALS AND METHODS}

This prospective study was carried out in the Department of Obstetrics and Gynecology at Krishna Institute of Medical Sciences, Karad.

All high-risk patients admitted for induction of labour or in the latent phase of labour were selected.

\section{Inclusion criteria:}

- Patient with gestational age at $>34$ weeks

- Cephalic presentation

- Intact membranes

- One or more antenatal high risk factor ${ }^{4,5}$

\section{High-risk factors include:}

- Pregnancy-induced hypertension

- Intrauterine growth retardation

- Overdue pregnancy

- Oligohydramnios

- Gestational Diabetes

- Heart disease ${ }^{6,7}$

\section{OBSERVATIONS AND RESULTS}

In the present study, 150 study subjects with high-risk pregnancy were studied using the electronic foetal monitor. The non-stress test was used primarily as an indicator for antepartum foetal surveillance. Results were analysed in terms of mode of delivery and perinatal outcome.

$64.67 \%$ of the patients belong to the age group of $20-24$ years as shown in this table.

$52.67 \%$ of the patients included in this study were primigravida.

$60.67 \%$ of the patients were of more than 40 weeks of gestational age( Table 1,2).

In this study, $83.33 \%$ of the patients were booked while $16.67 \%$ of the patients were unbooked (Table 3 ).

Commonest high-risk factor encountered in the present study was overdue pregnancy $(60.67 \%$, while overdue pregnancy alone as a high risk factor was present in $46.67 \%$ of the patients.
Table 6: Distribution of study samples according to Number of high-risk factors and non-stress test result

\begin{tabular}{lcccccc}
$\begin{array}{l}\text { No. of high- } \\
\text { risk factors }\end{array}$ & \multicolumn{2}{c}{ Normal } & \multicolumn{2}{c}{ Suspicious } & \multicolumn{2}{c}{ Abnormal } \\
& No. & $\%$ & No. & $\%$ & No. & $\%$ \\
\hline 1 & 69 & $60 \%$ & 34 & $29.57 \%$ & 12 & $10.43 \%$ \\
2 & 15 & $48.39 \%$ & 9 & $29.03 \%$ & 7 & $22.58 \%$ \\
3 & 1 & $25 \%$ & 1 & $25 \%$ & 2 & $50 \%$ \\
\hline
\end{tabular}

As seen in Table 6, the percentage of the abnormal non-stress test increases with an increase in the number of high-risk factors, while the percentage of the normal non-stress test is highest with only one high-risk factor.

This table shows the results of the non-stress test in each high-risk group. Out of 150 patients, $85(56.67 \%)$ non-stress test was normal, $44(29.33 \%)$ were suspicious and 21 (14\%) had an abnormal non-stress test.

$80 \%$ of patients with normal non-stress test delivered vaginally while only $50 \%$ and $28.57 \%$ with suspicious and abnormal non-stress test delivered vaginally, respectively. $71.43 \%$ of patients with abnormal non-stress test underwent a lower segment caesarean section. None of the patients had instrumental delivery (Table 4).

$17(20 \%)$ patients out of 85 patients with normal non-stress test required caesarean section while $22(50 \%)$ patients out of 44 and $16(76.19 \%)$ patients out of 21 patients with abnormal non-stress test required caesarean section.

Stillbirth was $9.52 \%$ and neonatal death was $9.52 \%$ in the abnormal non-stress test group. There were no stillbirth and $4.55 \%$ neonatal deaths in the suspicious non-stress test group. No stillbirth and neonatal death were observed in the normal non-stress test group (Table 5).

\section{DISCUSSION}

Obstetricians have long searched for methods of antepartum evaluation of foetus that would be noninvasive, accurate and yield immediately available results.

Over the past fifteen years, great strides have been made in antepartum foetal surveillance. The tests for foetal surveillance are being gradually improvised and modified to make them more sensitive and specific to permit the identification of those foetus requiring intervention, to prevent death or damage and allowing us not to interfere with those pregnancies doing well, letting healthy fetuses go to term. ${ }^{8,9}$

The ideal test should be easy to perform, less expensive, repeatable without causing inconvenience to the patient. It should be specific and sensitive. It should not have any side effects and should not cause any harm to the mother or 
the baby. Nonstress test is one such test which fulfils most of these criteria and thus, it is now generally accepted as a clinically useful method for detecting uteroplacental insufficiency and foetus at risk during the antepartum period. ${ }^{10}$

In this study, 150 patients with high-risk pregnancies were evaluated. Many similar studies were carried out over years in various countries.

In the present study, overdue pregnancy was the commonest risk factor (46.67\%). Overdue pregnancy was also found to be commonest risk factors in studies of Iman Bano et al (30\%). Uchil et al (45.34\%), Keegan and Paul (51.7\%) and Patil et al (19.7\%). In studies by Jain et al (61.8\%) and Habeebullah et al (27\%), bad obstetric history was the commonest risk factor. ${ }^{11,12}$

Iman Bano et al included pregnancy with jaundice and foetal tachycardia in their study while Habeebullah et al included cardiac diseases during pregnancy and premature rupture of membranes in their study. Uchil included infertility, previous caesarean section, placenta praevia in their series. Factors like infertility, previous caesarean section, threatened preterm labour were not included in this study because they were less likely to affect uteroplacental circulation. ${ }^{13}$

The non-stress test was done in these patients after 34 weeks of pregnancy. Maximum and minimum gestational age in studies of various authors is shown in this table.

In the present study, patients below 34 weeks of gestational age were not included because of the following reasons. ${ }^{14}$

A] Before 34 weeks, the incidence of the non reactive nonstress test is more due to immature control of the cardiovascular system. Characteristics of the premature non-stress test are

i) Less variability.

ii) More number of foetal movements which are less powerful and of short duration.

iii) Short episodes of activity and quiescence in the foetus (about 10 min. each).

iv) Before 30 weeks, decelerations are more common than accelerations in response to foetal movements. These decelerations are of short duration $(15-30 \mathrm{sec})$ with an amplitude of 15-30 bpm.

v) Foetal tachycardia. ${ }^{15,16}$

Due to these factors, interpretation of the non-stress test is very difficult.

B] Foetal survival could not be guaranteed because of less developed neonatal care units in our setup.

In the present study, the normal non-stress test was observed in $56.67 \%$ non-stress tests. $29.33 \%$ of the non stress test was suspicious and $14 \%$ of the non-stress test was abnormal. The result of the non stress test in the study by Rochard et al was similar to the present study.

\section{Mode of delivery concerning non-stress test results}

In the present study 68 patients $(80 \%)$ delivered vaginally while 17 patients $(20 \%)$ underwent LSCS. The indications of LSCS were the failure of induction in 6 patients $(35.29 \%)$, non-progress of labour in 5 patients $(29.41 \%)$, foetal distress in 3 patients $(17.65 \%)$ and thick meconium-stained liquor, DTA and cervical dystocia in 1 patient $(5.88 \%)$ each.

Percentage of vaginal delivery in the present study with normal non-stress test result was similar to that of study by Imam Bano et al. ${ }^{17}$

\section{Mode of delivery about the abnormal non- stress test:}

In the present study, only 6 patients $(28.57 \%)$ with abnormal non-stress test results delivered vaginally, while 15 patients (71.43\%) underwent LSCS. The indications of LSCS were foetal distress in 9 patients $(60 \%)$, failure of induction in 4 patients $(26.67 \%)$ and thick meconium-stained liquor in 2 patients $(13.33 \%)$.

Percentage of vaginal delivery and LSCS in the present study with abnormal non-stress test result was similar to that of study by Imam Bano et al.

\section{Suspicious non-stress test:}

In the present study, 22 patients ( $50 \%$ ) had a vaginal delivery and 22 patients $(50 \%)$ underwent LSCS out of a total of 44 patients with the suspicious non-stress test.

\section{Perinatal outcome about non-stress test re- sults}

No perinatal mortality was observed in patients with the normal non-stress test. Rochard et al, Imam Bano et al, Bahebullah et al, and Patil et al also had no perinatal mortality in their studies in patients with the normal non-stress test.

Though Keegan et al observed 7\% perinatal mortality in his study.

\section{Perinatal outcome about the abnormal non- stress test:}

Percentage of perinatal mortality in patients with the abnormal non-stress test was $19.04 \%$ in the present study while it was as high as $39.6 \%$ in the study by Rochard et al and lowest being $1.6 \%$ in the study by Patil et al.

In the present study, there were 2 neonatal deaths and 2 stillbirths in patients with an abnormal non-stress test.

\section{Stillbirth:}

One patient was having severe Pregnancy-induced hypertension with intrauterine growth retard at 38 weeks of gestation 
and LSCS was not considered due to very low expected birth weight. Patient delivered a stillborn child of 1020 grams.

Another patient having severe PIH with heart disease with IUGR at 35 weeks of gestation and keeping in mind the very low expected birth weight and risk of complications due to heart disease (severe mitral stenosis), the option of LSCS was not considered. Patient delivered a stillborn child of 1000 grams.

\section{Neonatal death}

There were 2 neonatal deaths in patients with the abnormal non-stress test. Both the patients underwent LSCS, one for foetal distress whose baby expired due to birth asphyxia while the other for thick meconium-stained liquor whose baby expired due to meconium aspiration syndrome.

Percentage of perinatal mortality with an abnormal nonstress test of the present study was $19.05 \%$. Imam Bano et al observed perinatal mortality of $50 \%$ and Rochard et al observed perinatal mortality of $39.6 \%$ with the abnormal nonstress test.

\section{Suspicious non-stress test}

In the present study, there was 2 neonatal death in patients with a suspicious non-stress test. One of the patients who had mild PIH with IUGR underwent LSCS for thick meconiumstained liquor and baby expired due to birth asphyxia and meconium aspiration syndrome. Another patient who had oligohydramnios had vaginal delivery and baby expired due to birth asphyxia and meconium aspiration syndrome.

\section{CONCLUSION}

Non-stress test is a simple, non-invasive, inexpensive test for antepartum foetal surveillance. It is easy to perform and causes no inconvenience or complications to the patient. The non-stress test should be performed for diagnostic purpose in the light of clinical circumstances.

Acknowledgement: Authors acknowledge the immense help received from the scholars whose articles are cited and included in references to this manuscript. The authors are also grateful to authors/editors/publishers of all those articles, journals and books from where the literature for this article has been reviewed and discussed.

Conflict of interest: Nothing to report.

Source of funding: Kimsdu Karad

\section{REFERENCES}

1. Baskett TF, Sandy E. False-negative oxytocin challenge tests. American Journal of Obstetrics \& Gynecology. 1975 Sep 1;123(1):106.

2. Baskett TF, Gray JH, Prewett SJ, Young LM, Allen AC. Antepartum fetal assessment using a fetal biophysical profile score. American journal of obstetrics and gynaecology. 1984 Mar $1 ; 148(5): 630-3$.

3. Rognerud Jensen $\mathrm{OH}$, Guimaraes MS. Prediction of the fetal outcome by Doppler examination and by the non-stress test. Acta obstetricia et Gynecologica Scandinavica. 1991 Jan 1;70(4-5):271-4.

4. Carter MC. Present - day performance qualities of cardiotocographs. BJOG: An International Journal of Obstetrics and Gynaecology. 1993 Mar;100:10-4.

5. Romano M, Iuppariello L, Ponsiglione AM, Improta G, Bifulco $\mathrm{P}$, Cesarelli M. Frequency and time-domain analysis of foetal heart rate variability with traditional indexes: a critical survey. Computational and mathematical methods in medicine. 2016 Oct;2016.

6. David H, Weaver JB, Pearson JF. Doppler ultrasound and fetal activity. Br Med J. 1975 Apr 12;2(5962):62-4.

7. Devoe LD. Clinical implications of prospective antepartum fetal heart rate testing. American journal of obstetrics and gynaecology. 1980 Aug 15;137(8):983-90.

8. Devoe LD, Jones CR. Nonstress test: evidence-based use in high-risk pregnancy. Clinical obstetrics and gynaecology. 2002 Dec 1;45(4):986-92.

9. Druzin ML, Gratacos J, Paul RH, Broussard P, McCart D, Smith M. Antepartum fetal heart rate testing: XII. The effect of manual manipulation of the fetus on the nonstress test. American journal of obstetrics and gynaecology. 1985 Jan 1;151(1):61-4.

10. Evertson LR, Gauthier RJ, Schifrin BS, Paul RH. Antepartum fetal heart rate testing: I. Evolution of the nonstress test. American Journal of Obstetrics and Gynecology. 1979 Jan 1;133(1):29-33.

11. Flynn AM, Kelly J. Evaluation of fetal wellbeing by antepartum fetal heart monitoring. Br Med J. 1977 Apr 9;1(6066):936-9.

12. Symonds EM. Litigation and the cardiotocograph. BJOG: An International Journal of Obstetrics \& Gynaecology. 1993 Mar;100:8-9.

13. Trierweiler MW, Freeman RK, James J. Baseline fetal heart rate characteristics as an indicator of the fetal status during the antepartum period. American Journal of Obstetrics and Gynecology. 1976 Jul 1;125(5):618-23.

14. Tushuizen PT, Stoot JE, Ubachs JM. Clinical experience in nonstressed antepartum cardiotocography. American Journal of Obstetrics and Gynecology. 1977 Jul 1;128(5):507-13.

15. Li T, Rhoads GG, Demissie K, Smulian J. The efficacy of the non - stress test in preventing fetal death in post - term pregnancy. Paediatric and perinatal epidemiology. 2001 Jul;15(3):26570.

16. Vintzileos AM, Campbell WA, Ingardia CJ, Nochimson DJ. The fetal biophysical profile and its predictive value. Obstetrics \& Gynecology. 1983 Sep 1;62(3):271-8.

17. Yeh SY, Forsythe A, Hon EH. Quantification of fetal heart beatto-beat interval differences. Obstetrics and Gynecology. 1973 Mar 1;41(3):355-63. 
Table 1: Distribution of study samples according to age-wise

$\begin{array}{lcc}\text { Age group } & \text { No. of patients } & \text { Percentage } \\ <20 & 9 & 6.00 \% \\ 20-24 & 97 & 64.67 \% \\ 25-29 & 38 & 25.33 \% \\ >29 & 6 & 4.00 \%\end{array}$

Table 2: Distribution of study samples according to Gravidawise distribution

\begin{tabular}{lcc} 
Gravida & No. of Patients & Percentage \\
Primi gravida & 79 & $52.67 \%$ \\
Second gravida & 50 & $33.33 \%$ \\
Third gravida & 18 & $12.00 \%$ \\
Fourth gravida and above & 3 & $2.00 \%$ \\
\hline
\end{tabular}

Table 3: Distribution of study samples according to Gestational age at the time of the non-stress test

\begin{tabular}{lcc} 
Gestational age (weeks) & No. of patients & Percentage \\
\hline $34-37$ & 14 & $9.33 \%$ \\
$37-40$ & 45 & $30.00 \%$ \\
$>40$ & 91 & $60.67 \%$ \\
\hline
\end{tabular}

Table 4: Distribution of study samples according to Booked / Unbooked

\begin{tabular}{lcc} 
Booked / Unbooked & No. of patients & Percentage \\
Booked & 125 & $83.33 \%$ \\
Unbooked & 25 & $16.67 \%$ \\
\hline
\end{tabular}

Table 5: Distribution of study samples according to High-risk factors

$\begin{array}{lcc}\text { High-risk factors } & \text { No. of Patients } & \text { Percentage } \\ \text { Overdue pregnancy } & 70 & 46.67 \% \\ \text { Mild PIH } & 23 & 15.33 \% \\ \begin{array}{l}\text { Oligohydroamnios } \\ \begin{array}{l}\text { Overdue pregnancy with } \\ \text { oligohydramnios }\end{array}\end{array} & 16 & 10.67 \% \\ \begin{array}{l}\text { Overdue pregnancy with } \\ \text { mild PIH }\end{array} & 5 & 9.33 \% \\ \begin{array}{l}\text { Severe PIH } \\ \text { Mild PIH with oligohydram- }\end{array} & 3 & 3.33 \% \\ \begin{array}{l}\text { nios } \\ \begin{array}{l}\text { Oligohydramnios with IUGR } \\ \text { Oligohydramnios with IUGR }\end{array}\end{array} & 3 & 2.67 \% \\ \text { with mild PIH } & 2 & 2.00 \% \\ \begin{array}{l}\text { Gestational diabetes } \\ \text { IUGR }\end{array} & 1 & 2.00 \% \\ \text { Mild PIH with IUGR } & 1 & 0.67 \% \\ & 1 & 0.67 \%\end{array}$

Table 5: (Continued)

\section{High-risk factors}

No. of Patients Percentage

Oligohydramnios with

o.67\%

severe PIH

Overdue pregnancy with

oligohydramnios with mild

$\mathrm{PIH}$

Overdue pregnancy with

severe PIH

Severe PIH with IUGR

Severe PIH with gestational diabetes

Severe PIH with IUGR with

heart disease

Severe PIH with oligohy-

dramnios

$\begin{array}{ll}1 & 0.67 \% \\ 1 & 0.67 \% \\ 1 & 0.67 \% \\ 1 & 0.67 \% \\ 1 & 0.67 \% \\ 1 & 0.67 \% \\ 1 & 0.67 \%\end{array}$

Table 7: Distribution of study samples according to Result of the non stress test in each high-risk group High-risk factors Normal Suspicious Abnormal

Overdue pregnancy

$\begin{array}{lll}45 & 22 & 3\end{array}$

Mild PIH

Oligohydroamnios

14

Overdue pregnancy with

oligohydramnios

Overdue pregnancy with

mild PIH

Severe PIH

Mild PIH with oligohy-

dramnios

Oligohydramnios with

IUGR

Oligohydramnios with

IUGR with mild PIH

Gestational diabetes

IUGR

Mild PIH with IUGR

Oligohydramnios with severe PIH

Overdue pregnancy with oligohydramnios with mild PIH

Overdue pregnancy with severe PIH

Severe PIH with IUGR

Severe PIH with gesta-

tional diabetes

Severe PIH with IUGR

with heart disease

Severe PIH with oligohy-

droamnios

Total

85

44

21 
Table 8: Distribution of study samples according to Mode of delivery about the non-stress test

\begin{tabular}{lcccccc} 
Mode of delivery & \multicolumn{2}{c}{ Normal } & \multicolumn{2}{c}{ Suspicious } & \multicolumn{2}{c}{ Abnormal } \\
& No. & $\%$ & No. & $\%$ & No. & $\%$ \\
Vaginal & 68 & $80 \%$ & 22 & $50 \%$ & 6 & $28.57 \%$ \\
LSCS & 17 & $20 \%$ & 22 & $50 \%$ & 15 & $71.43 \%$ \\
Total & 85 & & 44 & & 21 & \\
\hline
\end{tabular}

Table 9: Distribution of study samples according to Indication of lower segment caesarean section.

\begin{tabular}{lccc} 
Indication of LSCS & Normal & Suspicious & Abnormal \\
Foetal distress & 3 & 5 & 9 \\
Failure of induction & 6 & 4 & 4 \\
Non progress of labour & 5 & 5 & 0 \\
Thick meconium & 1 & 5 & 2 \\
Deep transverse arrest & 1 & 1 & 0 \\
Cervical dystocia & 1 & 0 & 0 \\
Non progress with thick meconium & 0 & 1 & 0 \\
Thick meconium with foetal distress & 0 & 1 & 0 \\
Total & 17 & 22 & 16 \\
\hline
\end{tabular}

Table 1o: Distribution of study samples according to Perinatal outcome about the non-stress test

\begin{tabular}{lcccccc} 
Perinatal & \multicolumn{2}{c}{ Normal } & \multicolumn{2}{c}{ Suspicious } & \multicolumn{2}{c}{ Abnormal } \\
outcome & No. & $\%$ & No. & $\%$ & No. & $\%$ \\
Good foetal outcome & 83 & $97.65 \%$ & 34 & $77.27 \%$ & 10 & $47.62 \%$ \\
NICU admission & 2 & $2.35 \%$ & 8 & $18.18 \%$ & 7 & $33 \cdot 33 \%$ \\
Neonatal death & 0 & - & 2 & $4.55 \%$ & 2 & $9.52 \%$ \\
Still birth & 0 & - & 0 & - & 2 & $9.52 \%$ \\
Total & 85 & & 44 & & 21 & \\
\hline
\end{tabular}

